\title{
A New Method to Find the Wiener Index of Hypergraphs
}

\author{
Yalan Li $\mathbb{D}^{1}$ and Bo Deng $\mathbb{D}^{2}$ \\ ${ }^{1}$ School of Computer, Qinghai Normal University, Xining 810001, China \\ ${ }^{2}$ School of Mathematics and Statistics, Qinghai Normal University, Xining 810001, China
}

Correspondence should be addressed to Bo Deng; dengbo450@163.com

Received 24 March 2020; Accepted 2 May 2020; Published 11 June 2020

Academic Editor: Florentino Borondo

Copyright (c) 2020 Yalan Li and Bo Deng. This is an open access article distributed under the Creative Commons Attribution License, which permits unrestricted use, distribution, and reproduction in any medium, provided the original work is properly cited.

The Wiener index is defined as the summation of distances between all pairs of vertices in a graph or in a hypergraph. Both models-graph-theoretical and hypergraph-theoretical-are used in mathematical chemistry for quantitatively studying physical and chemical properties of classical and nonclassical organic compounds. In this paper, we consider relationships between hypertrees and trees and hypercycles and cycles with respect to their Wiener indices.

\section{Introduction}

Let $G$ be a simple connected graph with vertex set $V(G)$ and edge set $E(G)$. For $u, v \in V(G)$, let $d_{G}(u, v)$ be the distance between the vertices $u$ and $v$ in $G$, and let $W_{G}(u)=\sum_{v \in V(G)} d_{G}(u, v)$ be the sum of distances between $u$ and all other vertices of $G$. The Wiener index of $G$ is defined as follows [1]:

$$
W(G)=\sum_{u, v \in V(G)} d_{G}(u, v)=\frac{1}{2} \sum_{u \in V(G)} W_{G}(u) .
$$

Let $H$ be a connected hypergraph with vertex set $V(H)$ and edge set $E(H)$, where $V(H)$ is nonempty and each edge $e \in E(H)$ is a nonempty subset of $V(H)$. If each edge contains exactly $k$ vertices, where $k \geq 2$, then a hypergraph $H$ is called $k$-uniform. It is obvious that a 2 -uniform hypergraph $H$ corresponds to an ordinary graph. The degree $d_{H}(v)$ of a vertex $v \in V(H)$ is the number of edges of $H$ which contains the vertex $v$. The order of $H$ is the cardinality of its vertex set $V(H)$.

For $u, v \in V(H)$, a path from $u$ to $v$ in $H$ is defined to be a sequence of distinct vertices and edges $\left(v_{0}, e_{1}, v_{1}, \ldots, v_{p-1}, e_{p}, v_{p}\right)$ such that $v_{i-1}, v_{i} \in e_{i}$ for any $i=1, \ldots, p$, where $v_{0}=u$ and $v_{p}=v$. For any $u, v \in V(H)$, if there exits a path from $u$ to $v$, then $H$ is connected. A $k$-uniform hypergraph $H$ with $V(H)=\left\{v_{1}, \ldots, v_{n}\right\}$ and
$E(H)=\left\{e_{1}, \ldots, e_{m}\right\}, \quad$ where $\quad e_{i}=\left\{v_{(i-1)(k-1)+1}, \ldots\right.$, $\left.v_{(i-1)(k-1)+k}\right\}, i=1, \ldots, m$, is called a $k$-uniform loose path and denoted by $P_{n, k}$. A hypercycle $\mathscr{C}_{p}$ of length $p$ in $H$ is a sequence $\left(v_{0}, e_{1}, v_{1}, \ldots, v_{p-1}, e_{p}, v_{p}\right), p \geq 2$, of distinct vertices (with the sole exception that $v_{0} \equiv v_{p}$ ) and distinct edges such that $v_{i-1}, v_{i} \in e_{i}, i=1, \ldots, p$. A hypertree is a connected hypergraph with no hypercycles. A $k$-uniform hypertree with $m$ edges has exactly $1+(k-1) m$ vertices. For a $k$-uniform hypertree $H$ of order $n$, if there is a disjoint partition $V(H)=\{u\} \cup V_{1} \cup \cdots \cup V_{m}$ of the vertex set $V(H)$ such that $\left|V_{1}\right|=\cdots=\left|V_{m}\right|=k-1$, and $E(H)=$ $\left\{\{u\} \cup V_{i}: 1 \leq i \leq m\right\}$, then $H$ is called a $k$-uniform hyperstar centered at $u$ and denoted by $S_{n, k}$. In particular, $S_{1, k}$ is a hypergraph with a single vertex, and $S_{k, k}$ is a hypergraph with a single edge.

Let $H$ be a $k$-uniform hypergraph with vertex set $V(H)=\left\{v_{1}, \ldots, v_{n}\right\}$. For $u, v \in V(H)$, the distance between $u$ and $v$ in $H$ is denoted by $d_{H}(u, v)$. The Wiener index $W(H)$ is defined as the summation of distances among all unordered pairs of distinct vertices $u, v$ in $H$ :

$$
W(H)=\sum_{\{u, v\} \subseteq V(H)} d_{H}(u, v) .
$$

Especially, the summation of distances from the vertex $u$ to any other vertex is denoted by $W_{H}(u)=\sum_{v \in V(H)} d_{H}(u, v)$. Obviously, we have $W(H)=(1 / 2) \sum_{u \in V(H)} W_{H}(u)$. 
Hypergraph theory has found applications in chemistry [2-7], showing a higher accuracy of molecular structure, that is, the higher accuracy of the model and the greater diversity of the behavior of its invariants. As an important descriptor of molecular structures, the Wiener index of a graph has many applications as well [8-13].

In this paper, we show relationships between Wiener indices for hypertrees and trees and for hypercycles and cycles with respect to some transformations. Let $\mathscr{T}$ be a $k$-uniform hypertree with at most two vertices whose degrees are not less than 2 in each edge of $\mathscr{T}$. The following formulas are proved for a $k$-uniform hypertree $\mathscr{T}$ and a tree $T$ of order $n$ obtained from $\mathscr{T}$ by transformations:

$$
W(\mathscr{T})=(k-1)^{2} W(T)-(n-1)\left(\begin{array}{c}
k-1 \\
2
\end{array}\right),
$$

and for a $k$-uniform hypercycle $\mathscr{C}_{n}$ and a cycle $C_{n}$ obtained from $\mathscr{C}$ by transformations:

$$
W\left(\mathscr{C}_{n}\right)= \begin{cases}(k-1)^{2} W\left(C_{n}\right)+n^{2}\left(\begin{array}{c}
k-1 \\
2
\end{array}\right), & \text { if } n \text { is odd } \\
(k-1)^{2} W\left(C_{n}\right)+n^{2}\left(\begin{array}{c}
k-1 \\
2
\end{array}\right)-\frac{n}{2}(k-2), & \text { if } n \text { is even. }\end{cases}
$$

\section{Hypertrees and Trees}

To prove (3), we need additional definitions and notations.

Let $\mathscr{T}$ be a $k$-uniform hypertree with at most two vertices whose degrees are not less than 2 in each edge of $\mathscr{T}$. Any two edges of $\mathscr{T}$ have at most one common vertex which is called a joint vertex; otherwise, it is called a nonjoint vertex.

We get a tree $T$ from the hypertree $\mathscr{T}$ as follows: (1) in $\mathscr{T}$, we pick a nonjoint vertex in each pendent edge and pick all joint vertices to form vertex set $V(T)$; (2) if two vertices of $V(T)$ are in the same edge of the hypertree $\mathscr{T}$, then we connect them with an edge in $T$. A 4-uniform hypertree $\mathscr{T}$ and its corresponding tree $T$ are shown in Figure 1.

The following notation is used in the rest of the paper. In $k$-uniform hypertree, the joint vertex is denoted by $b_{j}, j \geq 1$. The nonjoint vertex of each pendent edge $e_{i}$ in a hypertree is denoted by $a_{i}$. The nonjoint vertex in the nonpendent edge is denoted by $c_{i}$. Figure 2 illustrates the notation above. If $\mathrm{e}$ is a pendent edge, we use $V(\mathscr{T}) \backslash v(e) \backslash\{b\}$ to denote delete all vertices in edge $e$ except joint vertex $b$ from $V(\mathscr{T})$.

Since there are $k-1$ nonjoint vertices in each pendent edge and $k-2$ nonjoint vertices in a nonpendent edge, the number of vertices in the induced tree $T$ is $n$. The following equations hold for a hypertree $\mathscr{T}$ and induced tree $T$ :

$$
\begin{aligned}
|E(\mathscr{T})| & =|E(T)|=n-1, \\
W_{\mathscr{T}}\left(a_{i}\right)= & (k-1) W_{T}\left(a_{i}\right), \\
W_{\mathscr{T}}\left(b_{i}\right)= & (k-1) W_{T}\left(b_{i}\right), \\
2 W(\mathscr{T})= & (k-1)^{2} \sum_{a_{i} \in T} W_{T}\left(a_{i}\right)+(k-1) \sum_{b_{i} \in T} W_{T}\left(b_{i}\right) \\
& +(k-2) \sum_{c_{i} \in \mathscr{T}} W_{\mathscr{T}}\left(c_{i}\right) .
\end{aligned}
$$

Lemma 1. Let $\mathscr{T}$ be a hypertree with joint vertices $b_{i}$ and nonjoint vertices $c_{i}$ in each nompendent edge $e_{i}, i=1, \ldots, s$. Then, the following equation holds:

$$
\begin{aligned}
\sum_{b_{i} \in V(\mathscr{T})} W_{\mathscr{T}}\left(b_{i}\right)-\sum_{c_{i} \in V(\mathscr{T})} W_{\mathscr{T}}\left(c_{i}\right) & =|E(\mathscr{T})|(k-1) \\
& =(n-1)(k-1) .
\end{aligned}
$$

Proof. By induction on the number of edges, let $|E(\mathscr{T})|=3$; then, either $\mathscr{T}$ is a $k$-uniform hyperstar $S_{|V(\mathscr{T})|, k}$ centered at vertex $b$ or $\mathscr{T}$ is a $k$-uniform loose path of length 3 .

If $\mathscr{T}$ is a $k$-uniform hyperstar $S_{|V(\mathscr{T})|, k}$ centered at vertex $b$, then there are no nonjoint vertices $c_{i} \in V(\mathscr{T})$, and hence we have

$$
\sum_{b_{i} \in V(\mathscr{T})} W_{\mathscr{T}}\left(b_{i}\right)-\sum_{c_{i} \in V(\mathscr{T})} W_{\mathscr{T}}\left(c_{i}\right)=W_{\mathscr{T}}(b)=3(k-1) .
$$

If $\mathscr{T}$ is a $k$-uniform loose path of length 3 , then $\mathscr{T}$ has two joint vertices $b_{1}$ and $b_{2}$. Choosing one nonjoint vertex $c_{2} \in e_{2}$, by (5), we have

$$
\begin{aligned}
W_{\mathscr{T}}\left(b_{1}\right)+W_{\mathscr{T}}\left(b_{2}\right)-W_{\mathscr{T}}\left(c_{2}\right)= & 4(k-1)+4(k-1) \\
& -5(k-1)=3(k-1) .
\end{aligned}
$$

Let $|E(\mathscr{T})|>3$, then we choose any pendent edge $e \in E(\mathscr{T})$ connecting to joint vertex $b$, and consider $\mathscr{T}^{\prime}=(V(\mathscr{T}) \backslash v(e) \backslash\{b\}, E(\mathscr{T}) \backslash e)$. We also choose one nonjoint vertex $c_{i}(i=1,2, \ldots, r)$ from nonpendent edge on the left of $b$. It can be seen that there is a joint vertex $b_{i}$ for each $c_{i}$ in the same edge. Then, it has the similar case for the right side of $b$ (see Figure 3 ). 


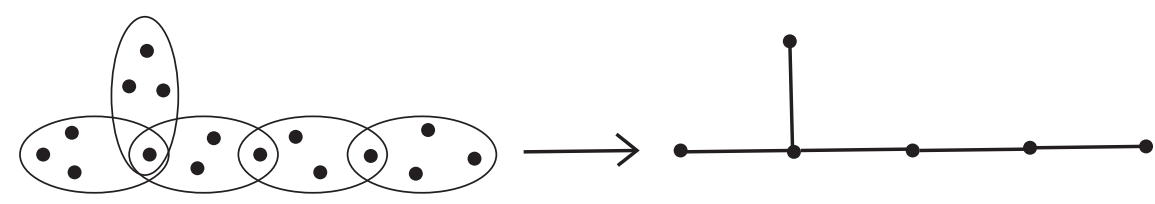

Figure 1: The 4-uniform hypertree $\mathscr{T}$ and the induced tree $T$.
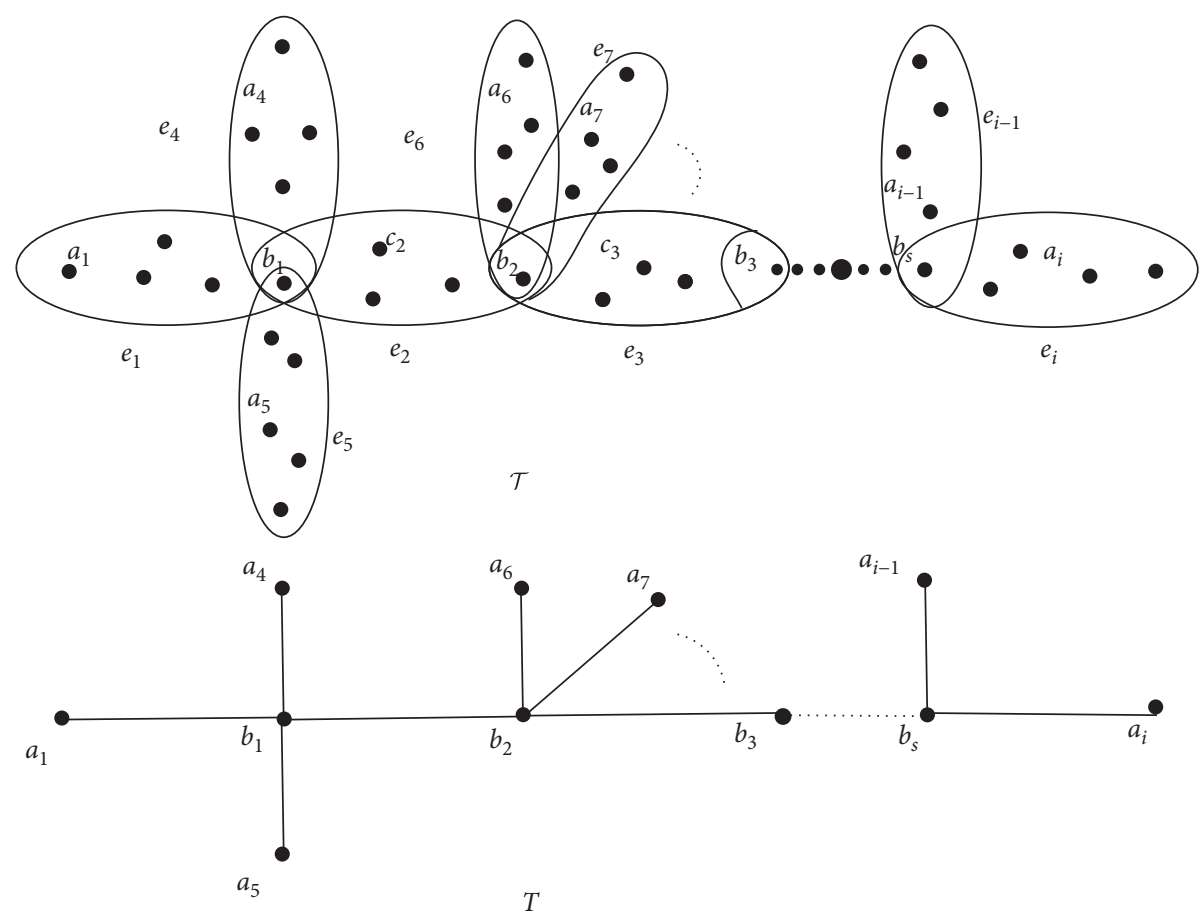

Figure 2: The $k$-uniform hypertree $\mathscr{T}$ and the induced tree $T$.

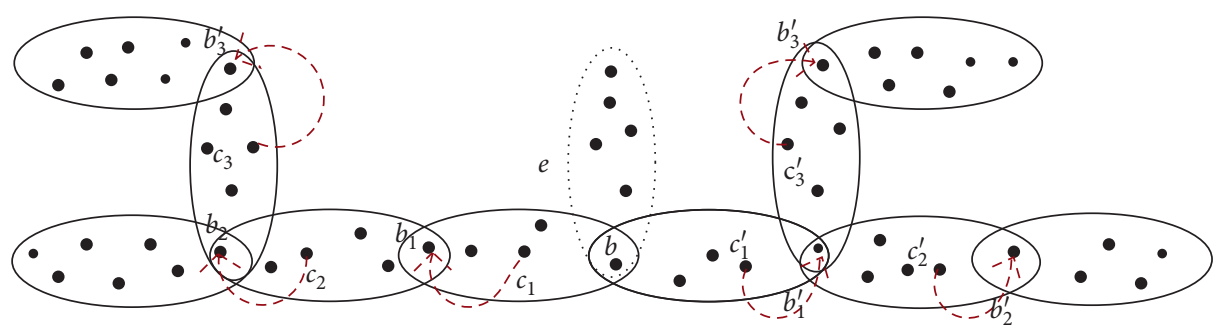

Figure 3: A $k$-uniform hypertrees $\mathscr{T}$.

Since the vertices $c_{i}$ and $b_{i}$ belong to the same edge in $\mathscr{T}$ and $\mathscr{T}^{\prime}$, they have the same increasing quality $\Delta(i)$ for their Wiener indices such that $W_{\mathscr{T}}\left(b_{i}\right)=W_{\mathscr{T}^{\prime}}\left(b_{i}\right)+\Delta(i)$ and
$W_{\mathscr{T}}\left(c_{i}\right)=W_{\mathcal{T}^{\prime}}\left(c_{i}\right)+\Delta(i)$. By the inductive hypothesis, we have

$$
\sum_{b_{i} \in V\left(\mathscr{T}^{\prime}\right)} W_{\mathscr{T}^{\prime}}\left(b_{i}\right)-\sum_{c_{i} \in V\left(\mathscr{T}^{\prime}\right)} W_{\mathscr{T}^{\prime}}\left(c_{i}\right)=\left|E\left(\mathscr{T}^{\prime}\right)\right|(k-1)=(|E(\mathscr{T})|-1)(k-1) .
$$


Thus, we obtain

$$
\begin{aligned}
& \sum_{b_{i} \in V(\mathscr{T})} W_{\mathscr{T}}\left(b_{i}\right)-\sum_{c_{i} \in V(\mathscr{T})} W_{\mathscr{T}}\left(c_{i}\right) \\
& =\sum_{b_{i} \in V(\mathscr{T}), b_{i} \neq b} W_{\mathscr{T}}\left(b_{i}\right)+W_{\mathscr{T}}(b)-\sum_{c_{i} \in V(\mathscr{T})} W_{\mathscr{T}}\left(c_{i}\right) \\
& =\sum_{b_{i} \in V\left(\mathscr{T}^{\prime}\right), b_{i} \neq b} W_{\mathscr{T}^{\prime}}\left(b_{i}\right)+\sum_{b_{i} \in V\left(\mathscr{T}^{\prime}\right), b_{i} \neq b} \Delta(i)+\left(W_{\mathscr{T}^{\prime}}(b)+k-1\right)-\sum_{c_{i} \in V\left(\mathscr{T}^{\prime}\right)} W_{\mathscr{T}^{\prime}}\left(c_{i}\right)-\sum_{c_{i} \in V\left(\mathscr{T}^{\prime}\right)} \Delta(i) \\
& =\sum_{b_{i} \in V\left(\mathscr{T}^{\prime}\right)} W_{\mathscr{T}^{\prime}}\left(b_{i}\right)-\sum_{c_{i} \in V\left(\mathscr{T}^{\prime}\right)} W_{\mathscr{T}^{\prime}}\left(c_{i}\right)+k-1 \\
& =(|E(\mathscr{T})|-1)(k-1)+k-1 \\
& =|E(\mathscr{T})|(k-1) \\
& =|E(T)|(k-1) \\
& =(n-1)(k-1) .
\end{aligned}
$$

Theorem 1. Let $T$ be an n-vertex tree obtained from the hypertree $\mathscr{T}$. Then,

$$
W(\mathscr{T})=(k-1)^{2} W(T)-(n-1)\left(\begin{array}{c}
k-1 \\
2
\end{array}\right) .
$$

Proof. By the definition of the Wiener index of a hypergraph, we have

$$
\begin{aligned}
2 W(\mathscr{T}) & =(k-1)^{2} \sum_{a_{i} \in V(T)} W_{T}\left(a_{i}\right)+(k-1) \sum_{b_{i} \in V(T)} W_{T}\left(b_{i}\right)+(k-2) \sum_{c_{i} \in V(\mathscr{T})} W_{\mathscr{T}}\left(c_{i}\right) \\
& =(k-1)^{2}\left[\sum_{a_{i} \in V(T)} W_{T}\left(a_{i}\right)+\sum_{b_{i} \in V(T)} W_{T}\left(b_{i}\right)\right]-(k-1)(k-2) \sum_{b_{i} \in V(T)} W_{T}\left(b_{i}\right)+(k-2) \sum_{c_{i} \in V(\mathscr{T})} W_{\mathscr{T}}\left(c_{i}\right) \\
& =2(k-1)^{2} W(T)-(k-1)(k-2) \sum_{b_{i} \in V(T)} W_{T}\left(b_{i}\right)+(k-2) \sum_{c_{i} \in V(\mathscr{T})} W_{\mathscr{T}}\left(c_{i}\right) \\
& =2(k-1)^{2} W(T)-(k-2)\left[\sum_{b_{i} \in V(\mathscr{T})} W_{\mathscr{T}}\left(b_{i}\right)-\sum_{c_{i} \in V(\mathscr{T})} W_{\mathscr{T}}\left(c_{i}\right)\right] .
\end{aligned}
$$

Then,

$$
W\left(S_{t, k}\right)=(t-1)^{2}-\frac{(t-1)(k-2)}{2} .
$$

$$
\begin{aligned}
W(\mathscr{T})= & (k-1)^{2} W(T)-\frac{k-2}{2} \\
& \cdot\left[\sum_{b_{i} \in V(\mathscr{T})} W_{\mathscr{T}}\left(b_{i}\right)-\sum_{c_{i} \in V(\mathscr{T})} W_{\mathscr{T}}\left(c_{i}\right)\right] .
\end{aligned}
$$

Proof. By $W\left(S_{((t-1) /(k-1))+1}\right)=((t-1) /(k-1))^{2}$ and from Theorem 1, we have

$$
W\left(S_{t, k}\right)=(k-1)^{2} W\left(S_{((t-1) /(k-1))+1}\right)-\left(\frac{t-1}{k-1}+1-1\right)\left(\begin{array}{c}
k-1 \\
2
\end{array}\right)
$$

By Lemma 1, we get

$$
W(\mathscr{T})=(k-1)^{2} W(T)-(n-1)\left(\begin{array}{c}
k-1 \\
2
\end{array}\right) .
$$

$$
\begin{aligned}
& =(k-1)^{2}\left(\frac{t-1}{k-1}\right)^{2}-\left(\frac{t-1}{k-1}\right)\left(\begin{array}{c}
k-1 \\
2
\end{array}\right) \\
& =(t-1)^{2}-\frac{(t-1)(k-2)}{2} .
\end{aligned}
$$

Corollary 1. Let $S_{t, k}$ be a k-uniform hyperstar of order $t$. 
Corollary 2. Let $P_{t, k}$ be a k-uniform loose path of order $t$. Then,

$$
W\left(P_{t, k}\right)=(k-1)^{2}\left(\begin{array}{c}
((t-1) /(k-1))+2 \\
3
\end{array}\right)-\frac{(t-1)(k-2)}{2} .
$$

Proof. It follows from Theorem 1 and by $W\left(P_{n}\right)=\left(\begin{array}{c}n+1 \\ 3\end{array}\right)$.

\section{Hypercycles and Cycles}

Let us consider the hypercycle $\mathscr{C}_{n}$ with $n$ joint vertices $b_{i}$, $i=1, \ldots, n$. If $b_{i}$ and $b_{j}, i \neq j$, are in the same edge in $\mathscr{C}_{n}$, then we connect them with an edge and get an $n$-vertex cycle $C_{n}$ from the hypercycle $\mathscr{C}_{n}$. We denote by $a_{i}$ a nonjoint vertex in each edge $e_{i}, i=1, \ldots, n$. The following statement gives us the relationship between Wiener indices of $\mathscr{C}_{n}$ and $C_{n}$.
TABle 1: The Wiener index of $k$-uniform hypercycles and corresponding cycles.

\begin{tabular}{ll}
\hline$W\left(\mathscr{C}_{2}\right)=(k-1)^{2}+4\left(\begin{array}{c}k-1 \\
2\end{array}\right)-(k-2)$ & $W\left(C_{2}\right)=1$ \\
$W\left(\mathscr{C}_{3}\right)=3(k-1)^{2}+9\left(\begin{array}{c}k-1 \\
2\end{array}\right)$ & $W\left(C_{3}\right)=3$ \\
$W\left(\mathscr{C}_{4}\right)=8(k-1)^{2}+16\left(\begin{array}{c}k-1 \\
2\end{array}\right)-2(k-2)$ & $W\left(C_{4}\right)=8$ \\
$W\left(\mathscr{C}_{5}\right)=15(k-1)^{2}+25\left(\begin{array}{c}k-1 \\
2\end{array}\right)$ & $W\left(C_{5}\right)=15$ \\
$W\left(\mathscr{C}_{6}\right)=27(k-1)^{2}+36\left(\begin{array}{c}k-1 \\
2\end{array}\right)-3(k-2)$ & $W\left(C_{6}\right)=27$ \\
$W\left(\mathscr{C}_{7}\right)=42(k-1)^{2}+49\left(\begin{array}{c}k-1 \\
2\end{array}\right)$ & $W\left(C_{7}\right)=42$ \\
\hline
\end{tabular}

Theorem 2.

$$
W\left(\mathscr{C}_{n}\right)= \begin{cases}(k-1)^{2} W\left(C_{n}\right)+n^{2}\left(\begin{array}{c}
k-1 \\
2
\end{array}\right), & \text { if } n \text { is odd } \\
(k-1)^{2} W\left(C_{n}\right)+n^{2}\left(\begin{array}{c}
k-1 \\
2
\end{array}\right)-\frac{n}{2}(k-2), & \text { if } n \text { is even }\end{cases}
$$

Proof. If $n$ is odd, the following equations are obtained by the definition of Wiener index:

$$
\begin{aligned}
2 W\left(C_{n}\right) & =n\left(1+1+2+2+\cdots+\frac{n-1}{2}+\frac{n-1}{2}\right)=\frac{n^{3}-n}{4}, \\
W_{\mathscr{C}_{n}}\left(b_{i}\right) & =\frac{\left(n^{2}+2 n+1\right)(k-1)}{4}-\frac{n+1}{2}, \\
W_{\mathscr{C}_{n}}\left(a_{i}\right) & =\frac{\left(n^{2}+4 n-1\right)(k-1)}{4}-\frac{n+1}{2}, \\
W\left(\mathscr{C}_{n}\right) & =\frac{1}{2} n W\left(b_{i}\right)+\frac{1}{2} n(k-2) W\left(a_{i}\right) \\
& =\frac{k-1}{2} \frac{n^{3}-n}{4}+\frac{n\left(n^{2}+4 n-1\right)}{4} \frac{(k-1)(k-2)}{2} \\
& =\frac{k-1}{2} \frac{n^{3}-n}{4}+\left(\frac{n^{3}-n}{8}+\frac{n^{2}}{2}\right)(k-1)(k-2) \\
& =(k-1) W\left(C_{n}\right)+\left(W\left(C_{n}\right)+\frac{n^{2}}{2}\right)(k-1)(k-2) \\
& =(k-1)^{2} W\left(C_{n}\right)+n^{2}\left(\begin{array}{c}
k-1 \\
2
\end{array}\right) .
\end{aligned}
$$

If $n$ is even, then by (5) and (6) we have the following equations:

$$
\begin{aligned}
& 2 W\left(C_{n}\right)= n\left(1+1+2+2+\cdots+\frac{n-2}{2}+\frac{n-2}{2}+\frac{n}{2}\right)=\frac{n^{3}}{4}, \\
& W_{\mathscr{C}_{n}}\left(b_{i}\right)= \frac{\left(n^{2}+2 n\right)(k-1)}{4}-\frac{n}{2}, \\
& W_{\mathscr{C}_{n}}\left(a_{i}\right)= \frac{\left(n^{2}+4 n\right)(k-1)}{4}-\frac{n+2}{2}, \\
& W\left(\mathscr{C}_{n}\right)= \frac{1}{2} n W\left(b_{i}\right)+\frac{1}{2} n(k-2) W\left(a_{i}\right) \\
&= \frac{k-1}{2} \frac{n^{3}-4 n}{4}+\frac{\left(n^{3}+4 n^{2}\right)}{4} \frac{(k-1)(k-2)}{2}+\frac{n}{2} \\
&= \frac{k-1}{2}\left(2 W\left(C_{n}\right)-n\right)+\left(2 W\left(C_{n}\right)+n^{2}\right) \\
& \cdot \frac{(k-1)(k-2)}{2}+\frac{n}{2} \\
&=(k-1)^{2} W\left(C_{n}\right)+n^{2}(k-1)-\frac{n}{2}(k-2) . \\
& 2
\end{aligned}
$$


Table 1 presents the Wiener index for the $k$-uniform hypercycles $\mathscr{C}_{i}$ and their corresponding cycles $C_{i}$, $2 \leq i \leq 7$.

\section{Data Availability}

No data were used to support this study.

\section{Conflicts of Interest}

The authors Yalan Li and Bo Deng declare that there are no conflicts of interest regarding the publication of this paper.

\section{Acknowledgments}

This work was supported by the NSFQH (nos. 2018-ZJ925Q, 2019-ZJ-7012, 2017-ZJ-949Q, and 2017-ZJ-790) and NSFC (nos. 11701311 and 11801296).

\section{References}

[1] I. Gutman and O. E. Polansky, Mathematical Concepts in Organic Chemistry, Springer Verlag, Berlin, Germany, 1986.

[2] H. Guo, B. Zhou, and H. Lin, "The Wiener index of uniform hypergraphs," MATCH Communications in Mathematical and in Computer Chemistry, vol. 78, pp. 133-152, 2017.

[3] I. Gutman, E. V. Konstantinova, and V. A. Skorobogatov, "Molecular hypergraphs and Clar structural formulas of benzenoid hydrocarbons," ACH Models in Chemistry, vol. 136, pp. 539-548, 1999.

[4] E. V. Konstantinova and V. A. Skoroboratov, "Graph and hypergraph models of molecular structure: a comparative analysis of indices," Journal of Structural Chemistry, vol. 39, no. 6, pp. 958-966, 1998.

[5] E. V. Konstantinova and V. A. Skorobogatov, "Application of hypergraph theory in chemistry," Discrete Mathematics, vol. 235, no. 1-3, pp. 365-383, 2001.

[6] E. V. Konstantinova, Chemical Hypergraph Theory, Pohang University of Science and Technology, Pohang, South Korea, 2001.

[7] H. Lin and B. Zhou, "Distance spectral radius of uniform hypergraphs," Linear Algebra and Its Applications, vol. 506, pp. 564-578, 2016.

[8] A. A. Dobrynin, R. Entringer, and I. Gutman, "Wiener index of trees: theory and applications," Acta Applicandae Mathematicae, vol. 66, no. 3, pp. 211-249, 2001.

[9] J. K. Doyle and J. E. Graver, "Mean distance in a graph," Discrete Mathematics, vol. 17, no. 2, pp. 147-154, 1977.

[10] P. J. Hansen and P. C. Jurs, "Chemical applications of graph theory. Part I. Fundamentals and topological indices," Journal of Chemical Education, vol. 65, no. 7, pp. 574-580, 1988.

[11] H. Hosoya, "Topological index. A newly proposed quantity characterizing the topological nature of structural isomers of saturated hydrocarbons," Bulletin of the Chemical Society of Japan, vol. 44, no. 9, pp. 2332-2339, 1971.

[12] J. Plesník, "On the sum of all distances in a graph or digraph," Journal of Graph Theory, vol. 8, no. 1, pp. 1-21, 1984.

[13] D. H. Rouvray, "Predicting chemistry from topology," Scientific American, vol. 255, no. 3, pp. 40-47, 1986. 